\title{
Interleukin-10 promotes primary rat hepatic stellate cell senescence by upregulating the expression levels of p53 and p21
}

\author{
YUE-HONG HUANG ${ }^{1,2}$, MING-HUA CHEN ${ }^{1}$, QI-LAN GUO ${ }^{1}$, YUN-XIN CHEN ${ }^{1}$, \\ LI-JUAN ZHANG ${ }^{1}$, ZHI-XIN CHEN $^{1}$ and XIAO-ZHONG WANG ${ }^{1}$ \\ Departments of ${ }^{1}$ Gastroenterology and ${ }^{2}$ Geriatrics, Fujian Medical University Union Hospital, \\ Fuzhou, Fujian 350001, P.R. China
}

Received August 28, 2017; Accepted December 14, 2017

DOI: $10.3892 / \mathrm{mmr} .2018 .8592$

\begin{abstract}
Liver fibrosis is characterized by the excessive deposition of extracellular matrix (ECM) components, and activated hepatic stellate cells (HSCs) are a primary source of ECM. Several studies have revealed that the induction of HSC senescence may reduce liver fibrosis. The effect of interleukin-10 (IL-10) on the senescence of activated HSCs is not fully understood. Therefore, the present study examined its effects and potential mechanisms in activated primary rat HSCs. Collagenase perfusion and density gradient centrifugation methods were used to isolate rat HSCs. HSCs were identified by autofluorescence, Oil Red $\mathrm{O}$ staining and immunocytochemical analysis. Activated HSCs were treated with $0,10,20$ or $40 \mathrm{ng} / \mathrm{ml} \mathrm{IL-10} \mathrm{for} 24 \mathrm{~h}$. Senescence-associated $\beta$-galactosidase (SA- $\beta$-Gal) staining, flow cytometry analysis and a cell counting kit- 8 assay were performed to detect the senescence, apoptosis and viability of rat HSCs, respectively. Reverse transcription-quantitative polymerase chain reaction, western blot analysis and enzyme linked immunosorbent assays were used to detect the expression of senescence-associated proteins and cytokines. Freshly isolated rat HSCs exhibited a striking blue-green autofluorescence and HSC retinoid droplets were stained bright red by Oil Red O. Immunocytochemical analysis demonstrated the cytoplasmic expression of HSC markers desmin and $\alpha$-smooth muscle actin. The number of SA- $\beta$-Gal positive HSCs, the apoptotic rate and the expression levels of p53, p21 and tumor necrosis factor- $\alpha$ were significantly increased following IL-10 treatment. HSC viability and IL-6 and IL-8 expression levels were significantly decreased compared with the control group. In summary, primary rat HSCs were successfully isolated and IL-10 was demonstrated
\end{abstract}

Correspondence to: Professor Xiao-Zhong Wang, Department of Gastroenterology, Fujian Medical University Union Hospital, 29 Xingquan Road, Fuzhou, Fujian 350001, P.R. China

E-mail: drwangxz@sina.com

Key words: interleukin-10, hepatic stellate cells, senescence, p53, p21, cytokine to promote the senescence of activated primary rat HSCs through the upregulation of p53 and p21 expression.

\section{Introduction}

Liver fibrosis occurs in response to chronic liver injuries, including hepatitis $\mathrm{B}$ and $\mathrm{C}$, alcohol consumption, fatty liver disease, cholestasis and autoimmune hepatitis (1). Activated hepatic stellate cells (HSCs) are the primary source of extracellular matrix (ECM) in the liver. Inhibiting the activation and promoting the apoptosis of HSCs would be an ideal therapeutic strategy to limit liver fibrosis (2). In recent decades, studies have revealed that the senescence of activated HSCs reduces liver fibrosis. The senescent HSCs have reduced viability, downregulated ECM component production, increased levels of ECM-degrading enzymes and enhanced immune surveillance (3). This indicates that promoting the senescence of activated HSCs may be an effective strategy in liver fibrosis therapy.

Senescence is a cellular process that irreversibly arrests the proliferation of damaged cells and induces the secretion of the inflammatory mediators IL-6, IL-8 and TNF- $\alpha$ which are part of a larger senescence associated secretory phenotype (SASP) (4). Cytokines have various roles in the development and/or promotion of premature cellular senescence (5). IL-6 regulates premature cellular senescence by activating its downstream signal transducer and activator of transcription3 (STAT3) and chemokine IL-8 has been demonstrated to reinforce the senescence process of cells (6). The inflammatory cytokine TNF- $\alpha$ promotes the premature senescence of rat nucleus pulposus cells via the PI3K/Akt signaling pathway (7). Interleukin-10 (IL-10) is a cytokine that downregulates the pro-inflammatory response and has a modulatory effect on hepatic fibrogenesis (8). Previous studies have demonstrated that IL-10 attenuates carbon tetrachloride- and porcine serum-induced liver fibrosis $(9,10)$. However, to the best of the author's knowledge, the role of IL-10 in activating HSC senescence has not yet been reported. The aim of present study was to obtain high-purity and yield primary rat HSCs to evaluate the effects of IL-10 on the senescence of activated HSCs and to examine the underlying mechanisms. The present study may provide a new insight on anti-fibrotic therapy of IL-10. 


\section{Materials and methods}

Animals. Fifteen male Sprague-Dawley rats (12-16-week-old) were provided by the Shanghai Experimental Animal Center (Shanghai, China). The rats were housed at $22 \pm 2^{\circ} \mathrm{C}$ with $55 \pm 5 \%$ humidity and a $12 \mathrm{~h}$ alternating light/dark cycle. Rats had access to water and food ad libitum. The present study was approved by the Animal Care Committee of Fujian Medical University (Fuzhou, China) and experiments were performed in accordance with the Guidelines on Animal Experiments in Fujian Medical University (Fuzhou, China).

Isolation and culture of primary rat HSCs. Rat HSC isolation was performed as previously described (11). Rat livers were perfused in situ and HSCs were isolated by density gradient centrifugation. Rat livers were perfused via the portal vein with D-Hanks buffer (PYG-0079, Boster Biological Technology, Wuhan, China) and subsequently with $0.075 \%$ type I collagenase for $10 \mathrm{~min}$ (Worthington Biochemical Corporation, Lakewood, NJ, USA). Livers were further digested by shaking for $20 \mathrm{~min}$ at $200 \mathrm{rpm}$ and $37^{\circ} \mathrm{C}$. Cell suspension was filtered to remove undigested debris and Hanks buffer was added to make up the solution to $50 \mathrm{ml}$. This was centrifuged at $50 \mathrm{x} \mathrm{g}$ for $5 \mathrm{~min}$ at room temperature to remove residual hepatocytes. Supernatant was subsequently transferred to a clean $50 \mathrm{ml}$ tube and centrifuged at $500 \mathrm{x} \mathrm{g}$ for $5 \mathrm{~min}$ at $4^{\circ} \mathrm{C}$. Cell pellets were resuspended in 12\% Histodenz (Sigma-Aldrich; Merck KGaA, Darmstadt, Germany) and centrifuged by density gradient for HSC isolation. The newly isolated HSCs were seeded in 6-well plates $\left(2 \times 10^{6}\right.$ cells/well) and cultivated in Dulbecco's modified Eagle's medium (DMEM; Hyclone; GE Healthcare Life Sciences, Logan, UT, USA) supplemented with $20 \%$ fetal bovine serum (FBS; Hyclone; GE Healthcare Life Sciences) at $37^{\circ} \mathrm{C}$ in an environment containing $5 \% \mathrm{CO}_{2}$ for $48 \mathrm{~h}$. Following seeding, HSCs were cultured in DMEM (Hyclone; GE Healthcare Life Sciences) supplemented with 10\% FBS (Hyclone; GE Healthcare Life Sciences) and 1\% penicillin and $1 \%$ streptomycin.

\section{Identification of primary rat HSCs}

Cell viability and yield. An equal volume $(0.1 \mathrm{ml})$ of DMEM-suspended HSCs were mixed with $0.4 \%$ Trypan blue solution. An aliquot of the mixture was analyzed in a Neubauer chamber following $5 \mathrm{~min}$ at room temperature and viable and non-viable cells within four big squares in the four corners were counted under a phase contrast microscopy (magnification, x100) The cell viability (\%) was calculated as follows: Total viable cells (unstained)/total cells (stained and unstained) $\times 100 \%$.

Autofluorescence detection of HSCs. Primary HSCs were seeded in 6-well plates $\left(2 \times 10^{5}\right.$ cells/well $)$ and cultured in DMEM (Hyclone; GE Healthcare Life Sciences) supplemented with 10\% FBS (Hyclone; GE Healthcare Life Sciences). Vitamin A lipid droplet autofluorescence in primary rat HSCs was detected at a wavelength of $328 \mathrm{~nm}$ using a fluorescence microscope (magnification, x200; Nikon Corporation, Tokyo, Japan).

Oil Red $O$ staining. Cells were fixed in $4 \%$ paraformaldehyde for $15 \mathrm{~min}$ at room temperature, washed with ice cold PBS and stained with $0.3 \%$ oil red $\mathrm{O}$ solution for $10 \mathrm{~min}$ at the room temperature. Stained slides were washed and counterstained with hematoxylin Staining Solution (Beyotime Institute of Biotechnology, shanghai, China) for $30 \mathrm{sec}$ at room temperature and washed again in PBS. Positive cells were observed from ten random fields under a phase contrast microscope (magnification, $\mathrm{x} 200$ ).

Immunocytochemistry assay. Cells were fixed in $4 \%$ paraformaldehyde for $15 \mathrm{~min}$ at room temperature and washed twice with ice cold PBS, and then permeabilized with $0.3 \%$ Triton X-100 for $15 \mathrm{~min}$. Endogenous peroxidase was removed with $30 \mathrm{ml} / 1 \mathrm{H}_{2} \mathrm{O}_{2}$. They were incubated with mouse anti-rat $\alpha$-smooth muscle actin ( $\alpha$-SMA, 1:400, cat. no. BM0002; Boster Biological Technology) and rabbit anti-rat desmin (1:200, cat. no. BM0036; Boster Biological Technology) primary antibodies in a humidified chamber overnight at $4^{\circ} \mathrm{C}$. Cells were treated with instant Polink-2 plus ${ }^{\circledR}$ Polymer HRP Detection System (cat. no. PV-9001/PV-9002; ZSGB-BIO; OriGene Technologies, Inc.) and incubated in a buffer solution containing 3,3-diaminobenzidine tetrahydrochloride (DAB) to produce a brown reaction product. Finally, cells were counterstained with hematoxylin Staining Solution (C0107; Beyotime Institute of Biotechnology) dehydrated, plated on coverslips and visualized under a phase contrast microscopy (magnification, $\mathrm{x} 200$ ). Counting the total 200 cells on the cover glass, the purity of hepatic stellate cells $(\%)=$ Desmin positive cells/total (positive and negative) $\mathrm{x} 100 \%$. the degree of HSC activation $(\%)=$ a-SMA positive cells/total (positive and negative) $\mathrm{x} 100 \%$.

Primary rat HSCs culture protocol. Primary rat HSCs were seeded in 6-well plates $\left(2 \times 10^{5}\right.$ cells/well $)$ and cultured in DMEM (Hyclone; GE Healthcare Life Sciences) supplemented with 10\% FBS (Hyclone; GE Healthcare Life Sciences). When cells reached $60-70 \%$ confluence, the culture medium was replaced with serum-free DMEM for $24 \mathrm{~h}$ and subsequently divided into the control and IL-10 treatment groups. Control group HSCs were cultured with DMEM supplemented with $10 \%$ FBS, whereas cells in the IL-10 treatment group were cultured with increasing concentrations of IL-10 (10, 20 or $40 \mathrm{ng} / \mathrm{ml}$; PeproTech, Inc., Rocky Hill, NJ, USA) for a further $24 \mathrm{~h}$ at $37^{\circ} \mathrm{C}$.

Primary rat HSCs senescence analysis. The Senescence $\beta$-Galactosidase Staining kit (C0602; Beyotime Institute of Biotechnology) was used to detect the senescence-associated $\beta$-galactosidase (SA- $\beta$-Gal) activity of activated HSCs. Briefly, HSCs treated with or without IL-10 were fixed with the staining fixative for $15 \mathrm{~min}$ at room temperature, washed with PBS and stained overnight with X-Gal solution at $37^{\circ} \mathrm{C}$. Cells were washed twice with PBS and incubated with 4',6-diamidino-2-phenlindole dihydrochloride $(0.5 \mu \mathrm{g} / \mathrm{ml}$; Roche Diagnostics, Indianapolis, IN, USA) for $10 \mathrm{~min}$. SA- $\beta$-Gal-positive cells were detected in at least three fields with a phase contrast microscope (magnification, x200) using Image-Pro Plus software (version 6.0; Media Cybernetics, Inc., Rockville, MD, USA).

Cell viability assay. The Cell Counting kit-8 (CCK-8; Dojindo Molecular Technologies, Inc., Kumamoto, Japan) was used to examine cell viability. Primary rat HSCs were seeded in 96-well 
plates $\left(3 \times 10^{3}\right.$ cells/well) in DMEM (Hyclone; GE Healthcare Life Sciences) supplemented with 10\% FBS (Hyclone; GE Healthcare Life Sciences) for $24 \mathrm{~h}$ and subsequently cultured without serum for $16 \mathrm{~h}$ prior to culture with either DMEM supplemented with $10 \%$ FBS alone or with increasing concentrations of IL-10 for $24 \mathrm{~h}$. The medium was replaced with fresh DMEM and $10 \mu$ l CCK-8 was added to each well for $2 \mathrm{~h}$. The absorbency was determined with a microplate reader $\left(\right.$ Epoch $^{\mathrm{TM}}$; BioTek Instruments, Inc., Winooski, VT, USA) at a wavelength of $450 \mathrm{~nm}$. The absorbance values were normalized by subtracting the blank values obtained from untreated cells.

Apoptosis assay. The FITC Annexin V Apoptosis Detection kit (cat. no. 556547; BD Biosciences, San Jose, CA, USA) was used to analyze the apoptosis of activated primary rat HSCs. Activated primary rat HSCs following treatment with or without IL-10 were washed twice with cold PBS and suspended in $1 \mathrm{X}$ Binding Buffer at a concentration of $1 \times 10^{6}$ cells $/ \mathrm{ml}$. Subsequently, $100 \mu 1$ of the solution ( $1 \times 10^{5}$ cells) were added to $5 \mathrm{ml}$ culture and add $5 \mu \mathrm{l}$ of FITC Annexin V and $5 \mu \mathrm{l}$ PI for $15 \mathrm{~min}$ at room temperature in the dark, $400 \mu \mathrm{l}$ of $1 \mathrm{X}$ Binding Buffer were added to each tube and analyzed using a FACScan cytometer (BD Accuri C6; BD Biosciences).

ELISA assay. Primary rat HSCs were treated with or without IL-10 for $24 \mathrm{~h}$. Cell culture supernatant was collected and assayed for IL-6 (cat. no. R6000B), IL-8 (cat. no. D8000C) and tumour necrosis factor (TNF)- $\alpha$ (cat. no. RTA00) expression levels using the respective ELISA kits, according to the manufacturer's protocol (R\&B Systems, Minneapolis, MN, USA).

Reverse transcription-quantitative polymerase chain reaction (RT-qPCR). Total RNA was isolated from HSCs using TRIzol $^{\circledR}$ reagent (Invitrogen; Thermo Fisher Scientific, Inc., Waltham, MA, USA) and reverse transcribed to cDNA using a Prime Script Reverse Transcription System (Takara Biotechnology Co., Ltd., Dalian, China) according to the manufacturer's protocol. cDNA samples were amplified using the SYBR-Green master mix kit (Takara Biotechnology Co., Ltd.). The primer sequences used were as follows: p53 forward, 5'-CTCСТCTCCCCAGCAAAAG-3' and reverse, 5'-CCTGCTGTCTCCTGACTCCT-3'; p21 forward, 5'-TGT GGTAGTTGGAGCTGGTG-3' and reverse, 5'-TGACCTGCT GTGTCGAGAAT-3'; and $\beta$-actin forward, 5'-GGCATCCTG ACCCTGAAGTA-3' and reverse, 5'-AGGCATACAGGGACA ACACA-3'. The PCR cycling conditions were as follows: $95^{\circ} \mathrm{C}$ for $2 \mathrm{~min}$ and $45 \mathrm{cycles}$ of $95^{\circ} \mathrm{C}$ for $15 \mathrm{sec}, 63^{\circ} \mathrm{C}$ for $15 \mathrm{sec}$, $72^{\circ} \mathrm{C}$ for $20 \mathrm{sec}$. All procedures were performed in triplicate. Alterations in mRNA expression were calculated relative to the expression control $\beta$-actin using the $2^{-\Delta \Delta \mathrm{Cq}}$ method (12).

Western blot analysis. HSCs were washed twice with PBS and lysed with radioimmunoprecipitation assay lysis buffer (Beyotime Institute of Biotechnology) and blocked in a phenylmethylsulfonyl fluoride (Beyotime Institute of Biotechnology) and PhosSTOP (Roche Diagnostics) cocktail on ice. The supernatant was obtained by centrifugation at $12,000 \mathrm{x} \mathrm{g}$ for $15 \mathrm{~min}$ at $4^{\circ} \mathrm{C}$. Protein concentration was determined by the enhanced BCA protein assay kit (Beyotime Institute of Biotechnology). Equal amounts $(40 \mu \mathrm{g})$ of protein were separated on a 10\% SDS-polyacrylamide gel and transferred onto nitrocellulose membranes. Following blocking with 5\% skim milk (cat. no. 1172GR500; BioFroxx GmbH, Einhausen, Germany; www.neofroxx.com/en/home/) in Tris-buffered saline/Tween-20 for $1 \mathrm{~h}$ at room temperature, the membranes were incubated with the primary antibodies (p53 (cat. no. 2524, 1:1,000; CST Biological Reagents, Co., Ltd., Shanghai, China), GAPDH (cat. no. 2118, 1:1,000; CST Biological Reagents, Co., Ltd.), and p21 (cat. no. ab109199, 1:1,000; Abcam, Cambridge, UK) at $4^{\circ} \mathrm{C}$ overnight, followed by incubation with horseradish peroxidase-conjugated homologous secondary antibody (cat. no. ZB-2301/ZB2305, 1:3,000; ZSGB-BIO; OriGene Technologies, Inc., Beijing, China) for $1 \mathrm{~h}$ at room temperature. The signals were visualized using an enhanced chemiluminescence kit (Santa Cruz Biotechnology, Inc., Dallas, TX, USA). The band density was determined by densitometry, and quantified using ChemiDoc ${ }^{\mathrm{TM}}$ Touch Imageing System with Image $\mathrm{Lab}^{\mathrm{TM}}$ Touch Software 5.2 version. (Bio-Rad Laboratories, Inc., Hercules, CA, USA).

Statistical analysis. Data are expressed as the mean \pm standard error. Statistical analysis was performed with one-way analysis of variance followed by Tukey's Multiple Comparison Test, using SPSS software, version 13.0 (SPSS, Inc., Chicago, IL, USA). $\mathrm{P}<0.05$ was considered to indicate a statistically significant difference.

\section{Results}

Isolation and identification of primary rat HSCs. To examine the effects of IL-10 on primary rat HSC senescence, isolation and culture of the HSCs was conducted. The collagenase perfusion and density-gradient centrifugation method (13) was used to isolate a high-purity and high-yield of quiescent rat HSCs. These were visible as a thin white layer in the interface between the Histodenz solution and the overlay with DMEM following isolation from density-gradient centrifugation (Fig. 1A). A total of $2-5 \times 10^{7}$ cells/rat were harvested. Cell viability as detected by Trypan blue staining was $>95 \%$. Phase contrast microscopy revealed that the morphology of plated HSCs was round and bright (Fig. 1B).

A typical feature of HSCs is the presence of cytoplasmic, triacylglycerol rich droplets (14). In the present study, rat HSCs emitted a striking, rapidly fading blue-green autofluorescence when excited by ultraviolet light at $328 \mathrm{~nm}$ (Fig. 1C). Quiescent HSCs may be identified with the Oil Red O stain. The retinoid droplets of HSCs cultured for $72 \mathrm{~h}$ were observed to be bright red under the light microscope (Fig. 1D). HSCs were identified using desmin, a reliable HSC marker (15) and $\alpha$-SMA, an activated HSC marker. HSCs were cultured for 7 days and immunocytochemical analysis demonstrated that desmin (Fig. 1E) and $\alpha$-SMA (Fig. 1F) were expressed in the HSC cytoplasm. The purity of the isolated HSCs was 93\% and the degree of HSC activation was $96 \%$.

IL-10 promotes the senescence of primary rat HSCs in vitro. The most widely used assay for cell senescence is the histochemical detection of $\beta$-galactosidase activity at $\mathrm{pH} 6.0$, which is known as SA- $\beta-$ Gal (4). In the present study, the effect of 
A

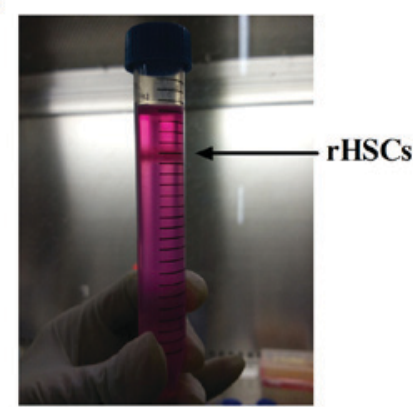

D

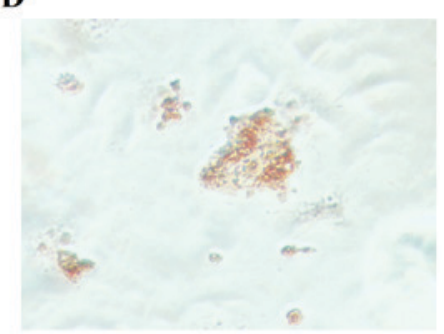

B

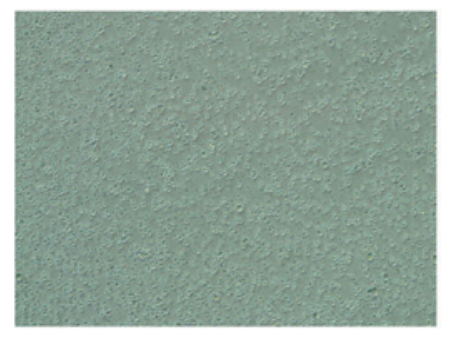

$\mathbf{E}$

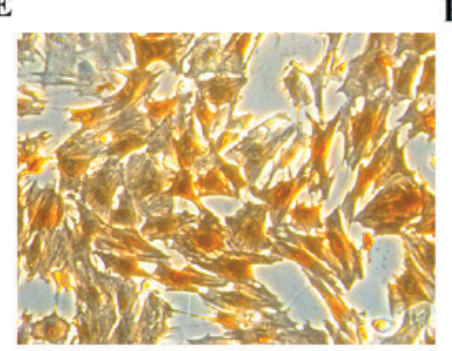

C

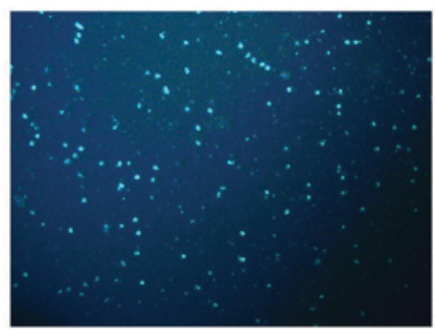

$\mathbf{F}$

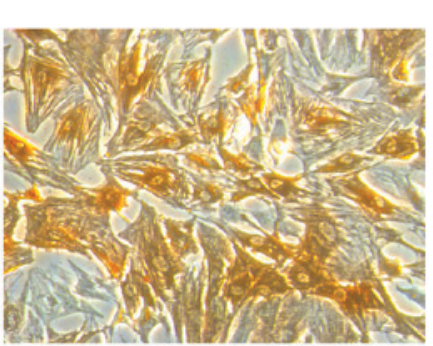

Figure 1. Identification of primary rHSCs. (A) rHSCs layered at the top of the Histodenz gradient and appeared as a white condensed band, as marked by the black arrow. (B) Light micrograph of rat HSCs at day 0 (magnification, x100). (C) Autofluorescence of vitamin A lipid droplets in rHSCs (magnification, x200). (D) Oil Red O staining (magnification, x200). (E) Desmin immunocytochemical staining (magnification, x200). (F) $\alpha$-smooth muscle actin immunocytochemical staining (magnification, x200). rHSCs, rat hepatic stellate cells.
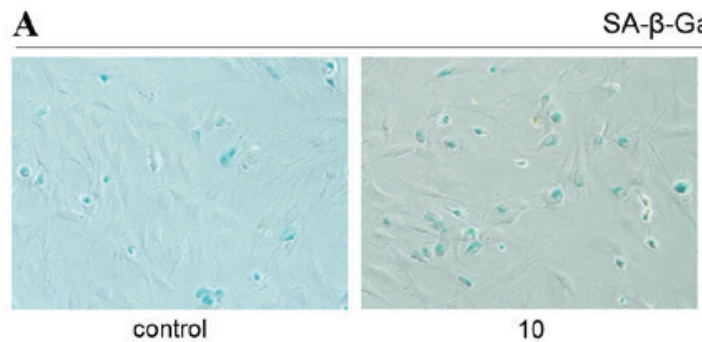

SA- $\beta$-Gal staining

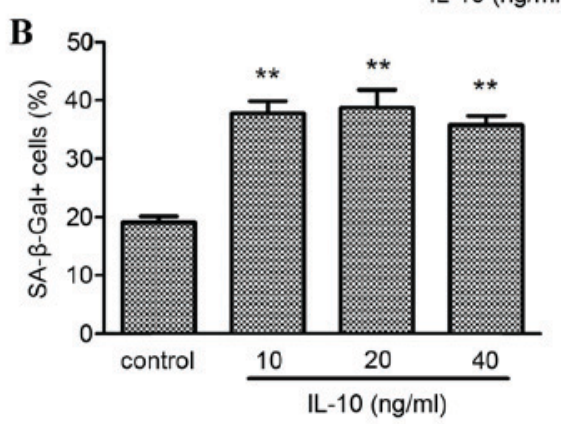

Figure 2. Effect of IL-10 on activated primary rat HSC senescence. Cells were incubated in serum-free medium for $24 \mathrm{~h}$ prior to incubation with increasing concentrations of IL-10 for $24 \mathrm{~h}$ and subsequent SA- $\beta$-Gal staining. (A) Positive staining was detected in all IL-10 treatment groups. (B) The number of SA- $\beta$-Gal positive HSCs were counted. "* $\mathrm{P}<0.01$ vs. control. HSC, hepatic stellate cell; IL-10, interleukin-10; SA- $\beta$-Gal, senescence-associated $\beta$-galactosidase.

IL-10 on the senescence of primary rat HSCs was examined by SA- $\beta$-Gal staining. Cells were incubated in serum-free medium for $24 \mathrm{~h}$, followed by incubation with different concentrations of IL-10 for a further $24 \mathrm{~h}$ prior to staining. The senescent primary rat HSCs cytoplasm was stained blue (Fig. 2A). The number of SA- $\beta$-Gal positive cells was significantly increased in HSCs treated in all IL-10 groups compared with the cells in the control group $(\mathrm{P}<0.01)$. The number of SA- $\beta-\mathrm{Gal}$ positive cells peaked in the $20 \mathrm{ng} / \mathrm{ml}$ IL-10 treatment group and subsequently decreased (Fig. 2B).
IL-10 promotes apoptosis and decreases the viability of activated primary rat HSCs. To investigate if IL-10 promoted primary rat HSCs senescence by decreasing viability or promoting apoptosis, flow cytometry analysis and a CCK-8 assay were performed, respectively. The apoptotic rate of primary rat HSCs increased significantly in the IL-10 treatment groups compared with the control group $(\mathrm{P}<0.001$; Fig. 3A and B) and HSC viability decreased significantly in the IL-10 treatment groups compared with the control group $(\mathrm{P}<0.01$; Fig. 3C). 


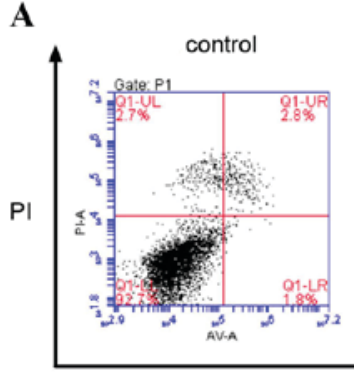

B

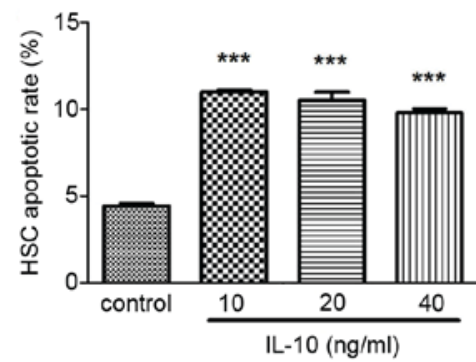

$\mathrm{IL}-10(\mathrm{ng} / \mathrm{ml})$

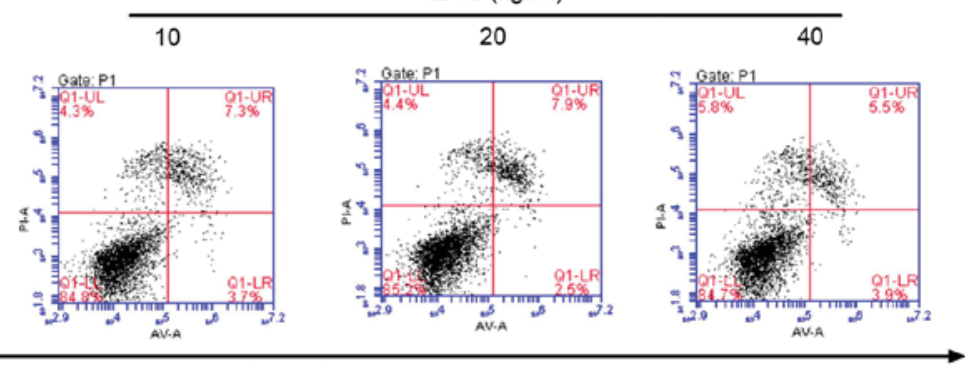

Annexin $\mathrm{V}$

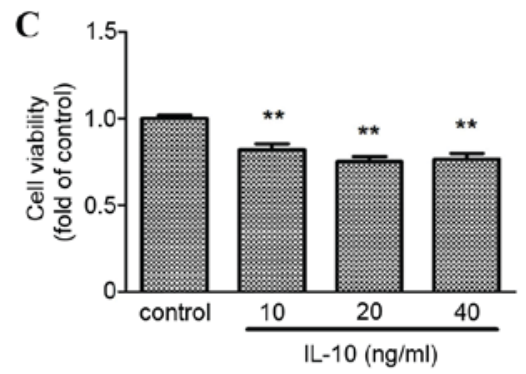

Figure 3. Effect of IL-10 on the apoptosis and viability of activated primary rat HSCs. Cells were incubated in serum-free medium for $24 \mathrm{~h}$ prior to incubation with increasing concentrations of IL-10 for 24 h, and subsequently flow cytometry analysis and Cell Counting kit-8 assay were conducted. (A) The apoptotic rate of rat HSCs was detected by flow cytometry analysis. (B) The relative quantification of apoptotic rate of rat HSCs treated with increasing concentrations of IL-10. (C) The viability of rat HSCs in different concentrations of IL-10 was determined by a cell counting kit- 8 assay ${ }^{* * *} \mathrm{P}<0.001,{ }^{* *} \mathrm{P}<0.01 \mathrm{vS}$. control. HSC, hepatic stellate cell; IL-10, interleukin-10; PI, propidium iodide.

IL-10 promotes the expression of senescence-associated proteins p53 and p21. p53 and p21 have a key role in cellular senescence, responding to a range of cellular damage signals (4). To examine the role of p53 and p21 in IL-10-induced senescence, p53 and p21 expression was detected in the absence or presence of IL-10 by RT-qPCR and western blot analysis. The expression of p53 mRNA was significantly increased in the IL-10 treatment groups compared with the control group ( $\mathrm{P}<0.05$; Fig. 4A), particularly in the $10 \mathrm{ng} / \mathrm{ml}$ group $(\mathrm{P}<0.001)$. p21 mRNA expression was also significantly increased in all IL-10 treatment groups $(\mathrm{P}<0.001$; Fig. 4B). Western blot analysis demonstrated that that p53 and p21 protein expression was increased in the IL-10 treatment groups (Fig. 4C). Relative quantification of protein expression revealed that p53 expression increased in a dose-dependent manner (Fig. 4D). Relative expression of p21 was highest in the 10 and $20 \mathrm{ng} / \mathrm{ml} \mathrm{IL-10} \mathrm{treatment} \mathrm{groups} \mathrm{(Fig.} \mathrm{4E).}$

IL-10 regulates the expression of senescence-associated inflammatory cytokines. Senescent cells secrete a number of inflammatory cytokines and chemokines that reinforce and propagate senescence in an autocrine and paracrine manner (4). The present study detected the expression of IL-6, IL-8 and TNF- $\alpha$ in HSCs treated with increasing concentrations of IL-10 for $24 \mathrm{~h}$. Compared with the control, IL-6 expression was markedly inhibited by IL-10 in a dose-dependent manner $(\mathrm{P}<0.001$; Fig. 5A). IL-8 levels were also significantly decreased in the 20 and 40 ng/ml IL-10 groups $(\mathrm{P}<0.001$; Fig. 5B). Conversely, TNF- $\alpha$ levels significantly increased in IL-10 treatment groups, particularly at $40 \mathrm{ng} / \mathrm{ml}(\mathrm{P}<0.001$; Fig. 5C).

\section{Discussion}

HSCs contribute to liver fibrosis and activated HSCs are considered the primary cellular target for anti-fibrotic therapies in all liver disease etiologies (16). Primary HSCs are a key tool in investigating the mechanisms underlying liver fibrosis and the efficacy of anti-fibrotic therapies. In the present study, primary rat HSCs were isolated by collagenase perfusion and density-gradient centrifugation and the effects of IL-10 on the senescence of activated HSCs was examined. Non-toxic and chemically near-inert components are ideal for density gradient centrifugation and it has been demonstrated that Histodenz is a suitable choice of medium (13). Therefore, Histodenz was used to isolate rat HSCs in the present study. A

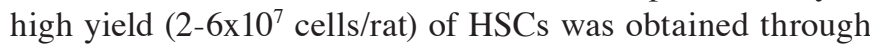
$12 \%$ Histodenz density-gradient centrifugation and desmin staining determined that the HSCs were $>90 \%$ pure. There are various steps that may be taken to increase the yield, purity and viability of HSCs. DMEM overlay must be added slowly, rats must weight $>500 \mathrm{~g}$, undigested debris after filter not to triturate and the time taken to isolate and digest the cells in vitro must be as short as possible (15). The present study demonstrated that freshly isolated HSCs stained positive for retinoid droplets and expressed desmin. Following culture and passage for 7 days, cells were activated and expressed $\alpha$-SMA. These results are consistent with previous reports (17) and lay the foundation for further study on the effect of IL-10 on HSC senescence.

The inhibition and removal of activated HSCs is a key strategy in the treatment of liver fibrosis (18). A previous study suggested that activated HSC senescence limits the extent of fibrosis following hepatic injury and that senescent HSCs lose the ability to produce collagen and proliferate (1). Cytokines may promote cellular senescence (5). IL-22 is a member of the IL-10 family that has been demonstrated to induce HSC senescence and reduce liver fibrosis in mice (19). Additionally, results of the author's previous study revealed that IL-10 attenuates porcine serum-induced liver fibrosis by inhibiting HSC 
A

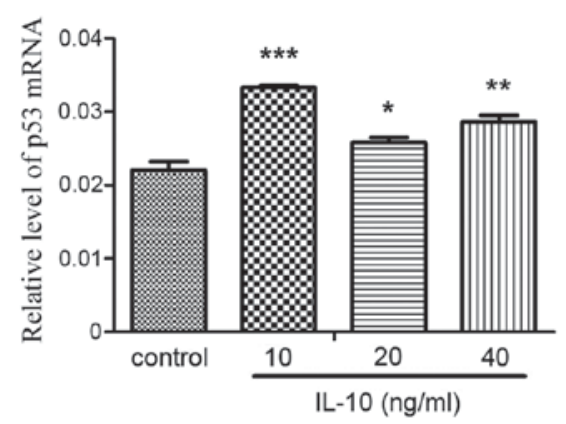

C

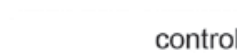

control

10
B

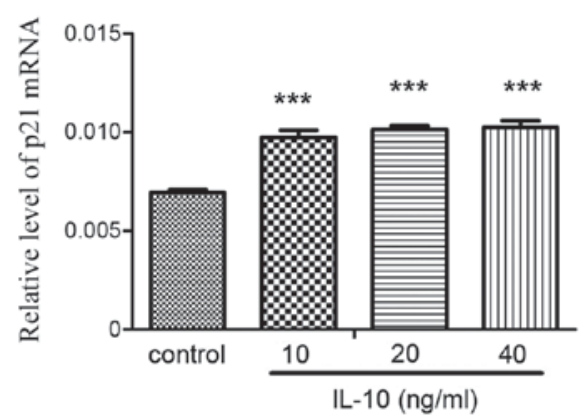

$\mathrm{IL}-10(\mathrm{ng} / \mathrm{ml})$

20

40

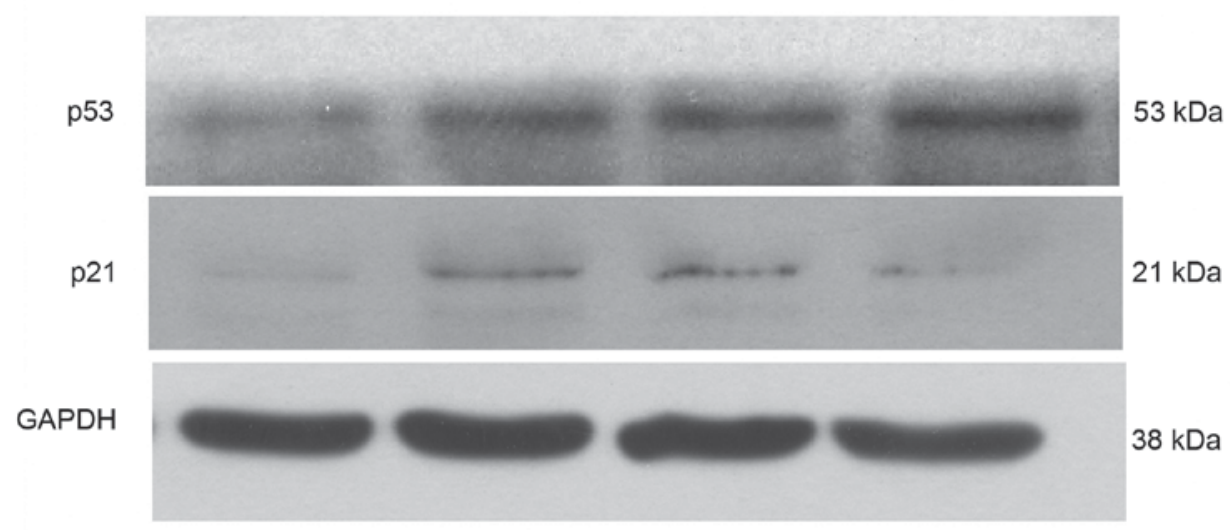

D

$\mathbf{E}$
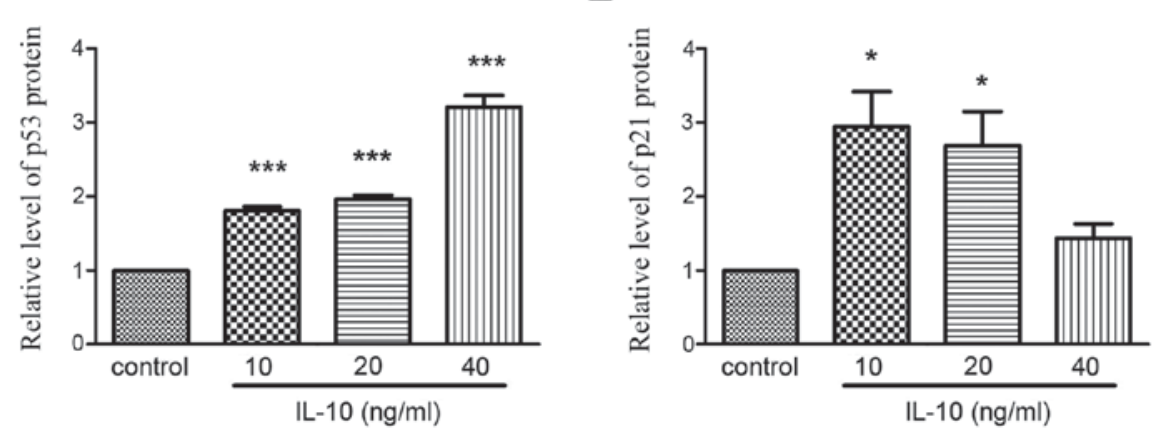

Figure 4. Effects of IL-10 on the expression of senescence associated proteins in activated primary rat HSCs. Cells were incubated in serum-free medium for $24 \mathrm{~h}$ prior to incubation with increasing concentrations of IL-10 for $24 \mathrm{~h}$ and subsequent RT-qPCR and western blot analysis. RT-qPCR analysis of (A) p53 and (B) p21 mRNA expression. (C) Western blot analysis of p53 and p21 protein expression Relative quantification of (D) p53 and (E) p21 protein expression. Data is expressed as the mean \pm standard deviation. ${ }^{*} \mathrm{P}<0.05,{ }^{* *} \mathrm{P}<0.01,{ }^{* * *} \mathrm{P}<0.001$ vs. control. HSC, hepatic stellate cell; IL-10, interleukin-10; RT-qPCR, reverse transcription-quantitative polymerase chain reaction.

A

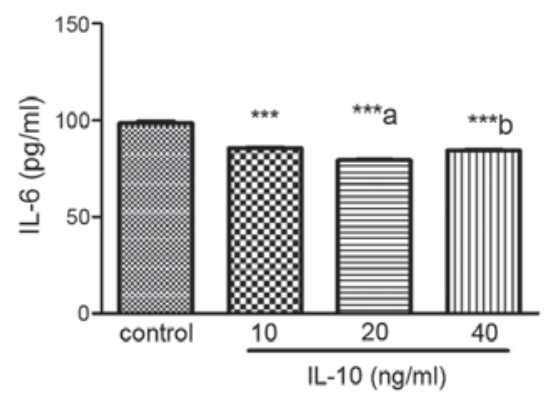

B

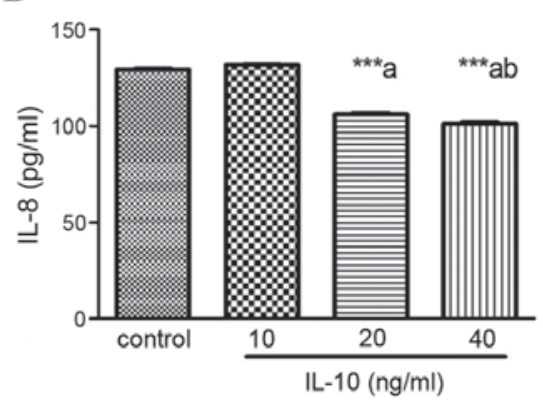

C

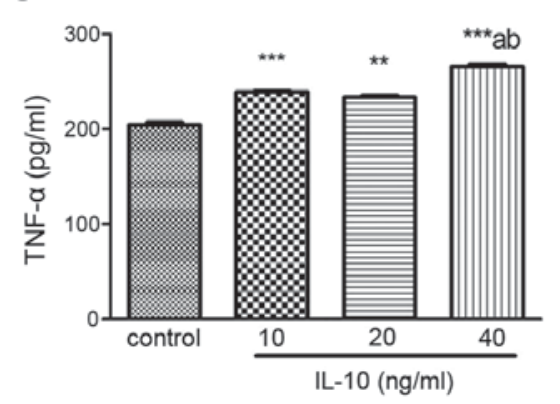

Figure 5. Effects of IL-10 on the expression of senescence associated cytokines. Cells were incubated in serum-free medium for 24 h prior to incubation with increasing concentrations of IL-10 for 24 h. Supernatant was collected and levels of (A) IL-6, (B) IL-8 and (C) TNF- $\alpha$ were detected by enzyme linked immunosorbent assays. ${ }^{* *} \mathrm{P}<0.01,{ }^{* * * *} \mathrm{P}<0.001$ vs. control group; ${ }^{a} \mathrm{P}<0.05$ vs. $10 \mathrm{ng} / \mathrm{ml}$; ${ }^{b} \mathrm{P}<0.05$ vs. $20 \mathrm{ng} / \mathrm{ml}$. IL, interleukin; TNF- $\alpha$, tumor necrosis factor- $\alpha$. 
activation (10). In a model of carbon tetrachloride-induced liver fibrosis, SA- $\beta$-Gal activity is detected in the fibrotic liver and SA- $\beta$-Gal positive cell accumulation in the area of fibrotic scar tissue occurs at an increased rate in IL-10 treated rats compared with untreated liver sections (Huang et al, unpublished data), suggesting that the anti-fibrotic effect of IL-10 may be associated with activated HSC senescence. The present study demonstrated that the senescence of HSCs increased following treatment with IL-10 in vitro.

Cellular senescence is a form of irreversible cell cycle arrest that occurs in response to cellular stressors, including DNA damage, oxidative stress, oncogene activation, chromatin disruption and telomere erosion (4). Senescent cells differ from other non-dividing cells. They have SA- $\beta$-Gal activity, an absence of proliferative markers and express tumor suppressors and cell cycle inhibitors (4). The most widely used assay for senescence is the histochemical detection of SA- $\beta-\mathrm{Gal}$ activity (4). The present study demonstrated that IL-10 treatment increased the activity of SA- $\beta$-Gal in HSCs. Senescent HSCs may inhibit HSC viability (20). The current study also revealed that IL-10 treatment not only limited HSC viability, however also promoted HSC apoptosis.

The primary mechanisms that control cell senescence are p53-dependent and retinoblastoma-dependent (5). The p53-dependent pathway has been recognized as one of the most important signal transduction pathways for the induction of cell senescence (4). p53 promotes senescence by transactivating genes including p21, microRNA-34 and B cell lymphoma- 2 associated $\mathrm{X}$, apoptosis regulator which result in cell cycle progression and viability inhibition (21). Previous studies have demonstrated that p53-p21 signaling pathway activation may induce HSC senescence and inhibit HSC viability $(18,20,22)$. p53 knockout mice exhibit severe hepatic fibrosis due to the inhibition of HSC senescence (23). The present study investigated if IL-10 induced HSC senescence through the upregulation of the p53-p21 signaling pathway. The results demonstrated that p53 and p21 mRNA and protein expression was significantly increased following IL-10 treatment, suggesting that the p53-p21 dependent pathway has an important role in IL-10-induced HSC senescence.

Cellular senescence not only decreases the viability of damaged cells, however also affects the microenvironment through the secretion of various pro-inflammatory cytokines, chemokines and proteases. This is termed the senescence-associated secretory phenotype (SASP) (24). SASP factors include IL- $1 \alpha$, IL-1 $\beta$, IL-6, IL- 8 and TNF- $\alpha(6,25)$. IL- 6 , IL- 8 and TNF- $\alpha$ have been demonstrated to reinforce the processes of senescence $(6,7)$. The upregulation of inflammatory cytokine expression makes HSCs susceptible to apoptosis through natural killer cell-mediated apoptosis, which limits the progression of fibrosis (3). To the best of the author's knowledge, the ability of IL-10 to promote activated HSC senescence though the upregulation of SASP has not yet been investigated. Therefore, the present study detected IL-6, IL-8, and TNF- $\alpha$ expression in activated HSCs following IL-10 treatment. The results revealed that IL-10 inhibited the expression of IL-6 and IL-8 and increased the expression of TNF- $\alpha$. Li et al (7) demonstrated that TNF- $\alpha$ may promote the premature senescence of rat nucleus pulposus cells. The mechanism of IL-6, IL-8 and TNF- $\alpha$ in IL-10-induced HSC senescence requires further study.
In conclusion, the present study demonstrated that primary rat HSCs may be successfully isolated at a high yield, viability and purity by the collagenase perfusion and density-gradient centrifugation method. IL-10 was revealed to promote the senescence of activated HSCs by upregulating the expression of p53 and p21.

\section{Acknowledgements}

The present study was supported by the National Natural Science Foundation of China (grant no. 81600486), the Fujian Province Nature Scientific Foundation of China (grant no. 2016J01462) and the Key Clinical Specialty Discipline Construction Program of Fujian and Nation, P.R. China.

\section{References}

1. Seki E and Brenner DA: Recent advancement of molecular mechanisms of liver fibrosis. J Hepatobiliary Pancreat Sci 22: 512-518, 2015.

2. Ramachandran P, Iredale JP and Fallowfield JA: Resolution of liver fibrosis: Basic mechanisms and clinical relevance. Semin Liver Dis 35: 119-131, 2015.

3. Krizhanovsky V, Yon M, Dickins RA, Hearn S, Simon J, Miething C, Yee H, Zender L and Lowe SW: Senescence of activated stellate cells limits liver fibrosis. Cell 134: 657-667, 2008.

4. Muñoz-Espín D and Serrano M: Cellular senescence: From physiology to pathology. Nat Rev Mol Cell Bio 15: 482-496, 2014.

5. Kojima H, Inoue T, Kunimoto $H$ and Nakajima K: IL-6-STAT3 signaling and premature senescence. JAKSTAT 2: e25763, 2014.

6. Kuilman T, Michaloglou C, Vredeveld LC, Douma S, van Doorn R, Desmet CJ, Aarden LA, Mooi WJ and Peeper DS: Oncogene-induced senescence relayed by an interleukindependent inflammatory network. Cell 133: 1019-1031, 2008.

7. Li P, Gan Y, Xu Y, Song L, Wang L, Ouyang B, Zhang C and Zhou Q: The inflammatory cytokine TNF- $\alpha$ promotes the premature senescence of rat nucleus pulposus cells via the PI3K/Akt signaling pathway. Sci Rep 7: 42938, 2017.

8. Hung KS, Lee TH, Chou WY, Wu CL, Cho CL, Lu CN, Jawan B and Wang CH: Interleukin-10 gene therapy reverses thioacetamide-induced liver fibrosis in mice. Biochem Biophys Res Commun 336: 324-331, 2005.

9. Huang YH, Shi MN, Zheng WD, Zhang LJ, Chen ZX and Wang XZ: Therapeutic effect of interleukin-10 on CCl4-induced hepatic fibrosis in rats. World J Gastroenterol 12: 1386-1391, 2006.

10. Huang YH, Chen YX, Zhang LJ, Chen ZX and Wang XZ: Hydrodynamics-based transfection of rat interleukin-10 gene attenuates porcine serum-induced liver fibrosis in rats by inhibiting the activation of hepatic stellate cells. Int J Mol Med 34: 677-686, 2014.

11. Shi MN, Huang YH, Zheng WD, Zhang LJ, Chen ZX and Wang XZ: Relationship between transforming growth factor beta1 and anti-fibrotic effect of interleukin-10. World J Gastroenterol 12: 2357-2362, 2006.

12. Livak KJ and Schmittgen TD: Analysis of relative gene expression data using real-time quantitative PCR and the 2(-Delta Delta C(T)) method. Methods 25: 402-408, 2001.

13. Weiskirchen R and Gressner AM: Isolation and culture of hepatic stellate cells. Methods Mol Med 117: 99-113, 2005.

14. Chang W, Yang M, Song L, Shen K, Wang H, Gao X, Li M, Niu W and Qin X: Isolation and culture of hepatic stellate cells from mouse liver. Acta Biochim Biophys Sin (Shanghai) 46: 291-298, 2014.

15. Mederacke I, Dapito DH, Affò S, Uchinami H and Schwabe RF: High-yield and high-purity isolation of hepatic stellate cells from normal and fibrotic mouse livers. Nat Protoc 10: 305-315, 2015.

16. Mederacke I, Hsu CC, Troeger JS, Huebener P, Mu X, Dapito DH, Pradere JP and Schwabe RF: Fate tracing reveals hepatic stellate cells as dominant contributors to liver fibrosis independent of its aetiology. Nat Commun 4: 2823, 2013.

17. Kim KH, Chen CC, Monzon RI and Lau LF: Matricellular protein CCN1 promotes regression of liver fibrosis through induction of cellular senescence in hepatic myofibroblasts. Mol Cell Biol 33: 2078-2090, 2013. 
18. Jin H, Lian N, Zhang F, Bian M, Chen X, Zhang C, Jia Y, Lu C, Hao M, Yao S, et al: Inhibition of YAP signaling contributes to senescence of hepatic stellate cells induced by tetramethylpyrazine. Eur J Pharm Sci 96: 323-333, 2017.

19. Kong X, Feng D, Wang H, Hong F, Bertola A, Wang FS and Gao B: Interleukin-22 induces hepatic stellate cell senescence and restricts liver fibrosis in mice. Hepatology 56: 1150-1159, 2012.

20. Chen J, Xu T, Zhu D, Wang J, Huang C, Lyu L, Hu B, Sun W and Duan Y: Egg antigen $\mathrm{p} 40$ of Schistosoma japonicum promotes senescence in activated hepatic stellate cells by activation of the STAT3/p53/p21 pathway. Cell Death Dis 7: e2315, 2016.

21. He L, He X, Lowe SW and Hannon GJ: microRNAs join the p53 network-another piece in the tumour-suppression puzzle. Nat Rev Cancer 7: 819-822, 2007.

22. Ahsan MK and Mehal WZ: Activation of adenosine receptor A2A increases HSC proliferation and inhibits death and senescence by down-regulation of p53 and Rb. Front Pharmacol 5: 69, 2014.
23. Kong D, Zhang F, Zhang Z, Lu Y and Zheng S: Clearance of activated stellate cells for hepatic fibrosis regression: Molecular basis and translational potential. Biomed Pharmacother 67: 246-250, 2013.

24. Zhang Z, Yao Z, Zhao S, Shao J, Chen A, Zhang F and Zheng S: Interaction between autophagy and senescence is required for dihydroartemisinin to alleviate liver fibrosis. Cell Death Dis 8: e2886, 2017.

25. Braumüller H, Wieder T, Brenner E, Aßmann S, Hahn M, Alkhaled M, Schilbach K, Essmann F, Kneilling M, Griessinger C, et al: T-helper-1-cell cytokines drive cancer into senescence. Nature 494: 361-365, 2013.

This work is licensed under a Creative Commons Attribution-NonCommercial-NoDerivatives 4.0 International (CC BY-NC-ND 4.0) License. 